\title{
Systems genetics-based drug discovery
}

\author{
H.-Y. Zhang \\ College of Informatics, Huazhong Agricultural University, Wuhan, P.R. China \\ e-mail: zhy630@mail.hzau.edu.cn
}

Key words: systems biology, medical genetics, drug discovery, gene networks, disease genes, gene predication

The past decade has witnessed unprecedented progress in biotechnology, particularly in omics technologies. However, these great technical advances have made limited contributions to the advancement of the pharmaceutical industry. It still requires \$2.6 billion and approximately 13.5 years on average for a new molecular entity (NME) drug to enter the market. Therefore, we urgently need methods that can efficiently synthesize and utilize biological big data to streamline the drug discovery pipeline.

In recent years, medical genetics has found important applications in drug discovery. However, most complex diseases are caused by multiple interacted causative factors; hence, agents that only hit single pathogenic factors are not very efficacious. Thus, to utilize medical genetic information more efficiently in drug development, we should aim at multiple disease genes, especially interacted pathogenic factors, to find potential drugs.

Systems genetics is a thriving area of study that aims to understand genetic interactions on a genome-wide scale. One of the representative algorithms in this field is the HotNet diffusion-oriented subnetworks (HotNet2) algorithm. HotNet2 is based on an insulated heat diffusion kernel algorithm that considers the heats (reflecting genetic importance) of individual genes as well as the topology of gene-gene interactions. This method can reveal functionally interacted genes within subnetworks, thus overcoming the limitations of traditional GWAS/PheWAS methods for elucidating disease pathogenesis.

In this study, we demonstrate that integrating HotNet2 calculation with GWAS/PheWAS can substantially promote the drug discovery efficiency. Besides, this method is also efficient to find drug combinations. 